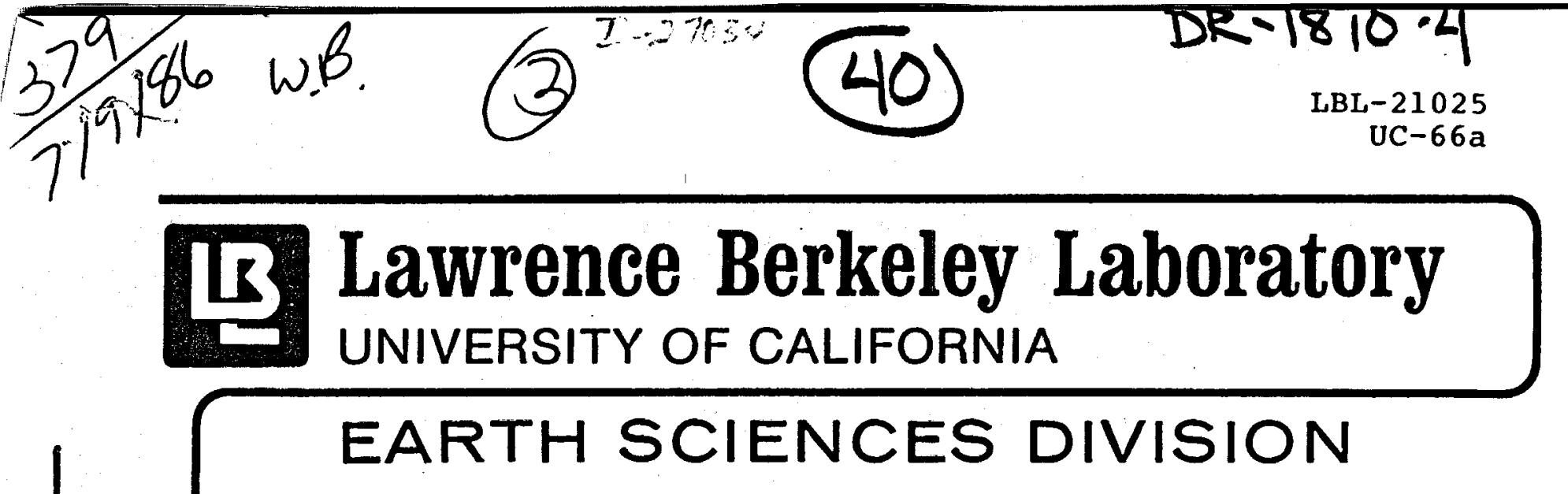

ON THE ACCURACY

OF THE MINC APPROXIMATION

C.H. Lai, K. Pruess and G.S. Bodvarsson

February 1986
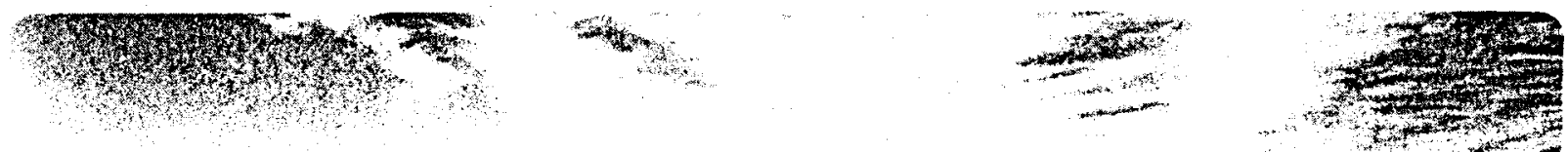

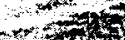

$\mathrm{s}^{2}+\mathrm{s}+\mathrm{x}$

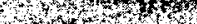

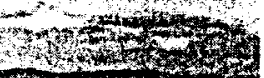

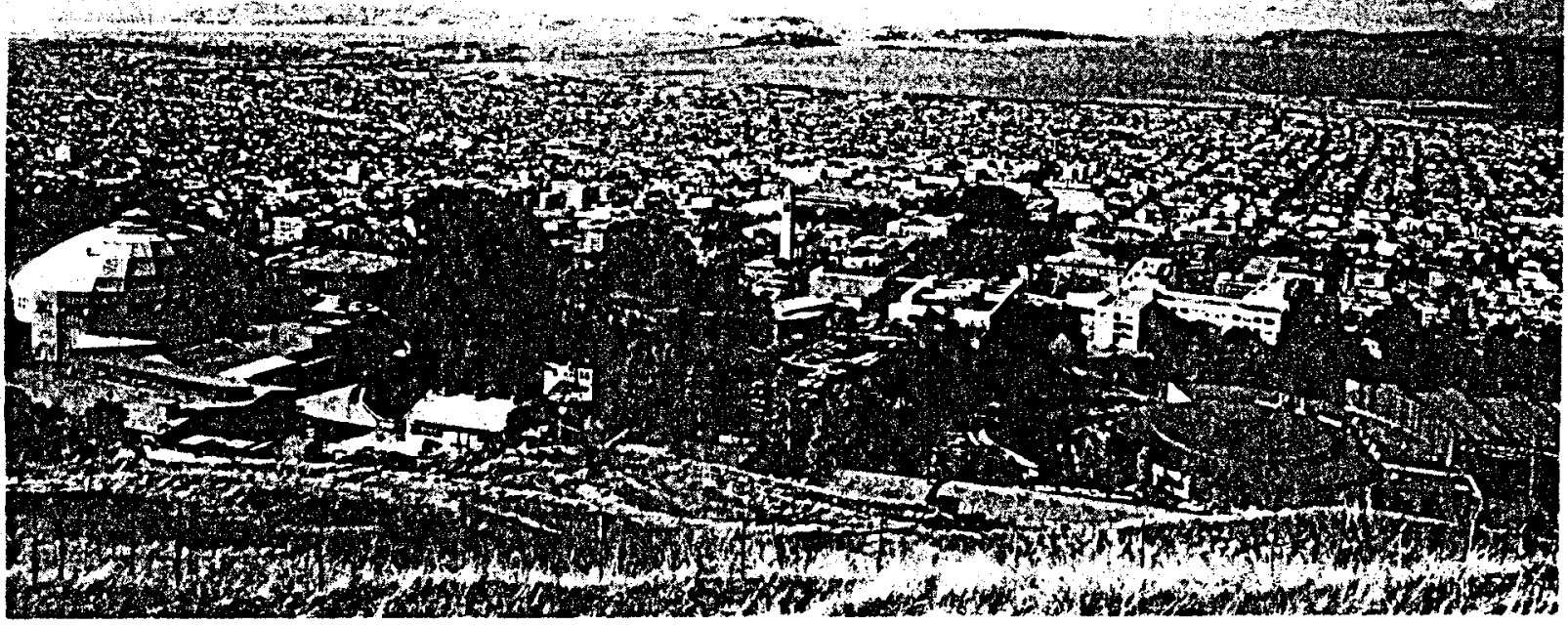

Prepared for the U.S. Department of Energy under Contract DE-AC03-76SF00098

DISTRIBUTIOH OF TIIS DOCUMENT IS UNLLATHEA 


\section{DISCLAIMER}

Portions of this document may be illegible in electronic image products. Images are produced from the best available original document. 


\section{DISCLAIMER}

This report was prepared as an account of work sponsored by an agency of the United States Government. Neither the United States Government nor any agency thereof, nor any of their employees, makes any warranty, express or implied, or assumes any legal liability or responsibility for the accuracy, completeness, or usefulness of any information, apparatus, product, or process disclosed, or represents that its use would not infringe privately owned rights. Reference herein to any specific commercial product, process, or service by trade name, trademark, manufacturer, or otherwise does not necessarily constitute or imply its endorsement, recommendation, or favoring by the United States Government or any agency thereof. The views and opinions of authors expressed herein do not necessarily state or reflect those of the United States Government or any agency thereof. 


\title{
ON THE ACCURACY OF THE MINO APPROXIMATION
}

\author{
C. H. Lai, K. Pruess and G. S. Bodvarsson
}

\section{MASTER}

Earth Sciences Division

Lawrence Berkeley Laboratory

University of California, Berkeley

Berkeley, CA 94720
LBL -21025

DE86 012753

\section{Introduction}

Modeling of transport process in fractured media is mathematically very difficult because of the complicated geometries and transport processes involved. Pruess and Narasimhan (1982) have developed an extension of the double-porosity method (Barenblatt, 1960; Warren and Root, 1963), referred to as method of "multiple interacting continua" (MINC), which can handle transient inter-porosity flow of fluid and heat in fractured porous media. The MINC approximation assumes that, due to high permeability and low storativity of the fractures, any changes of thermodynamic conditions in a fractured porous medium will propagate rapidly in the fracture network, while migrating only slowly into the low-permeability rock matrix blocks. Therefore, the changes of thermodynamic conditions in the rock matrix blocks will depend primarily on the distance to the nearest fracture. In light of this and by neglecting gravity effects, fluid and heat flow in the rock matrix blocks may be treated by a one-dimensional approximation. This concept is applicable to regular as well as irregular matrix blocks (Pruess and Karasaki, 1983).

In numerical simulations, the MINC method partitions rock matrix blocks in to sets of nested volume elements (Figures 1 and 2). Thus, the interactions between fractures and the rock matrix can be described by one-dimensional mass and energy conservation equations. When applicable, this approximation can save substantial amounts of 
computer time and storage in comparison to the more detailed discretization by conventional finite difference methods. However, the accuracy of the method employed needs to be tested and justified (Pruess et al., 1982). In general, mass and heat flow are not perpendicular to the fracture surfaces, especially near fracture intersections ("corners"), and hence can not strictly be considered one-dimensional. To study this "corner" effect, we have considered some idealized geometrical configurations and simple boundary conditions, for which exact solutions as well as solutions based on the MINC approximation are available in analytical and semi-analytical form.

\section{Case 1: Fluid Flow in a Porous Cube}

The test case considered is for isothermal, slightly compressible fluid flow in a porous cube (or, equivalently, heat conduction in an impermeable cube). A constant pressure, $P_{b}$, is maintained at the cube surfaces, and an initial pressure of zero is assumed everywhere. With the MINC approximation, fluid flow in a cube can be approximated by a one dimensional model, as shown in Figure 3. The basic model represents one-sixth of a cube, with the surface area for flow decreasing from $D^{2}(D$ is the side length of the cube) at the edges of the cube to zero in the center. Thus, the total mass flow at the cube surfaces will be six times that given by the one-dimensional model. This onedimensional approximation leads to a differential equation whose form is identical to the heat conduction equation in a system with spherical geometry. The dimensionless pressure and flow rate for this problem using the one-dimensional approximation is given by Carslaw and Jaeger (1959):

$$
P_{D}=\frac{P}{P_{b}}=1-\frac{D}{\pi z} \sum_{n=1}^{\infty} \frac{(-1)^{n}}{n} \sin \frac{2 n \pi z}{D} \exp \left\{-\frac{4 n^{2} \pi^{2} k t}{\phi \mu c D^{2}}\right\}
$$

and

$$
q_{D}=\frac{q \mu}{k P_{b}}=24 \sum_{n=1}^{\infty} \exp \left\{-\frac{n^{2} \pi^{2} k t}{\phi c \mu(D / 2)^{2}}\right\}
$$


The same problem can be solved exactly in three dimension. The dimensionless transient pressure and flow rate is given by (Carslaw and Jaeger, 1959):

$$
P_{D}=\frac{P}{P_{b}}=1-\frac{64}{\pi^{3}} \sum_{l=1}^{\infty} \sum_{m=1}^{\infty} \sum_{n=1}^{\infty} \frac{(-1)^{l+m+n-3}}{(2 l-1)(2 m-1)(2 n-1)} \cos \frac{(2 l-1) \pi x}{D}
$$

$$
\cos \frac{(2 l-1) \pi y}{D} \cos \frac{(2 l-1) \pi z}{D} \exp \left\{-\frac{k \pi^{2} t}{\phi \mu c D^{2}}\left[(2 l-1)^{2}+(2 m-1)^{2}+(2 n-1)^{2}\right]\right\}
$$

and

$$
\begin{aligned}
q_{D}=\frac{q \mu}{k P_{J} D} & =\frac{24 \times 64}{\pi^{4}} \sum_{l=1}^{\infty} \sum_{m=1}^{\infty} \sum_{l=1}^{\infty} \frac{1}{(2 l-1)^{2}(2 m-1)^{2}} \\
& \exp \left\{-\frac{\pi^{2}}{4}\left[(2 l-1)^{2}+(2 m-1)^{2}+(2 n-1)^{2}\right] \frac{k t}{\phi c \mu(D / 2)^{2}}\right\}
\end{aligned}
$$

In the above equations, $(x, y, z)$ - coordinates are measured from the center of the cube and parallel to the edges. The dimensionless pressures at a distance $z=0.3 D$ for the MINC approximation and for the exact solution are plotted versus dimensionless time in Figure 4. The figure clearly indicates that in the center of the plane $z=0.3 D$ (ie., for $x^{\prime}=\frac{x}{D}=0, y^{\prime}=\frac{y}{D}=0$ ) the pressures from the MINC approximation are somewhat higher than the exact pressures, but in the corner of that plane $\left(x^{\prime}=y^{\prime}=0.3\right)$ they are somewhat lower. The discrepancies are not large (about 10-15\%). What really matters, however, is not the detailed pressure distributions inside the cube, but the total (areally integrated) flow rate at the cube surface. Figure 5 shows that the flow rate at the cube surface using the MINC approximation agrees very well with the exact solution.

\section{Case 2: Fluid Flow in a Rectangular. Porous Slab}

To further test the MINC approximation, a comparison was made for fluid flow in two-dimensional rectangular matrix blocks, with side lengths $A$ and $B$ for different aspect ratios $\beta=A / B$. The same initial and boundary conditions are used as in case 1 . 
With the MINC approximation, the basic model (Figure 6) of a rectangle will be solved. The governing equation describing the mass conservation in the domain of the basic model can be expressed as:

$$
q S-\left\{q S+\frac{\partial}{\partial z}(q S) d z\right\}=\frac{\partial(S d z \phi \rho)}{\partial t}
$$

where $q$ is mass flow rate, $\phi$ is porosity, $\rho$ is fluid density, $t$ is time, and $S$ is the cross section surface area in the $z$ direction expressed as:

$$
S=4 z+A-B
$$

Substituting Eq. 6 and Darcy's law $\left(q=-\frac{\rho k}{\mu} \nabla P\right)$ into Eq. 5, the governing equation describing slightly compressible fluid flow in the domain of the basic model can be expressed as:

$$
\frac{\partial^{2} P}{\partial z^{2}}+\frac{1}{z+\frac{1}{4}(A-B)} \frac{\partial P}{\partial z}=\frac{\phi \mu c}{k} \frac{\partial P}{\partial t}
$$

where $c$ is fluid compressibility and $k$ is intrinsic permeability of the medium. The initial and boundary conditions are:

$$
\begin{gathered}
P(z, 0)=0 \\
P\left(\frac{B}{2}, t\right)=P_{b} \\
\left.\frac{\partial P}{\partial z}\right|_{z=0}=0
\end{gathered}
$$

In terms of dimensionless parameters, the governing equation and the initial and boundary conditions can be written as:

$$
\frac{\partial^{2} P_{D}}{\partial \eta^{2}}+\frac{1}{\eta} \frac{\partial P_{D}}{\partial \eta}=\frac{\partial P_{D}}{\partial \tau}
$$




$$
P_{D}=0
$$

$$
P_{D}\left(\eta=\frac{A+B}{4 B}, \tau\right)=1
$$

$$
\left.\frac{\partial P_{D}}{\partial \eta}\right|_{\eta=\frac{A-B}{A B}}=0
$$

where

$$
\begin{gathered}
P_{D}=\frac{P}{P_{b}} \\
\eta=\frac{z+1 / 4(A-B)}{B} \\
\tau=\frac{k t}{\phi \mu c B^{2}}
\end{gathered}
$$

In the Laplace domain, the solution of Eq. 7 subject to the given initial and boundary conditions is:

$$
\bar{P}_{D}=\frac{1}{p} \frac{K_{1}\left(\sqrt{p} \frac{A-B}{4 B}\right) I_{0}(\sqrt{p} \eta)+K_{0}(\sqrt{p} \eta) I_{1}\left(\sqrt{p} \frac{A-B}{4 B}\right)}{K_{1}\left(\sqrt{p} \frac{A-B}{4 B}\right) I_{0}\left(\sqrt{p} \frac{A+B}{4 B}\right)+K_{0}\left(\sqrt{p} \frac{A+B}{4 B}\right) I_{1}\left(\sqrt{p} \frac{A-B}{4 B}\right)}
$$

where $p$ is the Laplace parameter. The dimensionless mass flow rate at the surface of the rectangle can be obtained from Eq. 18 by evaluating the pressure gradient at the surface:

$$
\bar{q}_{D}=2\left(\frac{A}{B}+1\right) \frac{1}{\sqrt{p}} \frac{K_{1}\left(\sqrt{p} \frac{A-B}{4 B}\right) I_{1}\left(\sqrt{p} \frac{A+B}{4 B}\right)-K_{1}\left(\sqrt{p} \frac{A+B}{4 B}\right) I_{1}\left(\sqrt{p} \frac{A-B}{4 B}\right)}{K_{1}\left(\sqrt{p} \frac{A-B}{4 B}\right) I_{0}\left(\sqrt{p} \frac{A+B}{4 B}\right)+K_{0}\left(\sqrt{p} \frac{A+B}{4 B}\right) I_{1}\left(\sqrt{p} \frac{A-B}{4 B}\right)}
$$

where $q_{D}$ is $\frac{q \mu}{k P_{b}}$. In this study, the solution for dimensionless flow rate in real space is obtained by numerical inversion of Eq. 19 (Stehfest, 1970). The exact solution for this 
two-dimensional problem is given by Carslaw and Jaeger (1959):

$$
\begin{aligned}
& P_{D}=1-\frac{16}{\pi^{2}} \sum_{l=1}^{\infty} \sum_{m=1}^{\infty} \frac{(-1)^{l+m-2}}{(2 l-1)(2 m-1)} \cos \frac{(2 l-1) \pi x}{A} \cos \frac{(2 m-1) \pi y}{B} \\
& \quad \exp \left\{-\frac{k \pi^{2} t}{\phi \mu c}\left[\frac{(2 l-1)^{2}}{A^{2}}+\frac{(2 m-1)^{2}}{B^{2}}\right]\right\}
\end{aligned}
$$

and

$$
q_{D}=\frac{64}{\pi^{2}} \sum_{l=1}^{\infty} \sum_{m=1}^{\infty}\left\{\frac{A}{B} \frac{1}{(2 l-1)^{2}}+\frac{B}{A} \frac{1}{(2 m-1)^{2}}\right\} \exp \left\{-\frac{k \pi^{2} t}{\phi \mu c}\left[\frac{(2 l-1)^{2}}{A^{2}}+\frac{(2 m-1)^{2}}{B^{2}}\right]\right\}
$$

Figure 7 shows that the dimensionless mass flow rate across the surface of the rectangle obtained from the MINC approximation for different aspect ratios $(\beta)$ compares well with the exact solution. The agreement becomes close when the aspect ratio is increased, because this will diminish the "corner" effects neglected by the MINC approximation.

\section{Conclusions}

The method of "multiple interacting continua" is based on the assumption that changes in thermodynamic conditions of rock matrix blocks are primarily controlled by the distance from the nearest fracture. We have evaluated the accuracy of this assumption for regularly shaped (cubic and rectangular) rock blocks with uniform initial conditions, which are subjected to a step change in boundary conditions on the surface. Our results show that pressures (or temperatures) predicted from the MINC approximation may deviate from the exact solutions by as much as $10-15 \%$ at certain points within the blocks. However, when fluid (or heat) flow rates are integrated over the entire block surface, MINC-approximation and exact solution agree to better than $1 \%$. This indicates that the MINC approximation can accurately represent transient inter-porosity flow in fractured porous media, provided that matrix blocks are indeed subjected to nearly uniform boundary conditions at all times. 


\section{References:}

1. Barenblatt, G. I., Zheltov, Iu. P. and Kochina, I. N., 1960, Basic Concepts in the Theory of Seepage of Homogeneous Liquids in Fissured Rocks, J. Appl. Math. Mech., Vol. 24, 1286-1303.

2. Carslaw, H. S. and Jaeger, J. C., 1959, Conduction of Heat in Solids (2nd Ed.), London, Oxford University Press, 510pp.

3. Pruess, K. and Narasimhan, T. N., 1982, A Practical Method for Modeling Fluid and Heat Flow in Fractured Porous Media, Paper Presented at the 6th Annual Meeting of SPE, New Orleans, Louisiana, Jan. 31-Feb. 2.

4. Pruess, K. et al., 1982, Advances in Numerical Modeling of Fractured Geothermal Reservoirs, LBL-15500, 129-132.

5. Pruess, K. and Karasaki, K., 1983, Proximity Functions for Modeling Fluid and Heat Flow in Reservoirs with Stochastic Fracture Distributions, Presented at 8th Workshop on Geothermal Reservoir Engineering, Stanford University, Stanford, CA.

6. Stehfest, H., 1970, Numerical Inversion of Laplace Transforms, Communications of ACM, Vol. 13, 44-49:

7. Warren, J. E. and Root, P. J., 1963, The Behavior of Naturally Fractured Reservoirs, Soc. Pet. Eng. J., 245-225, Trans., AIME 228. 


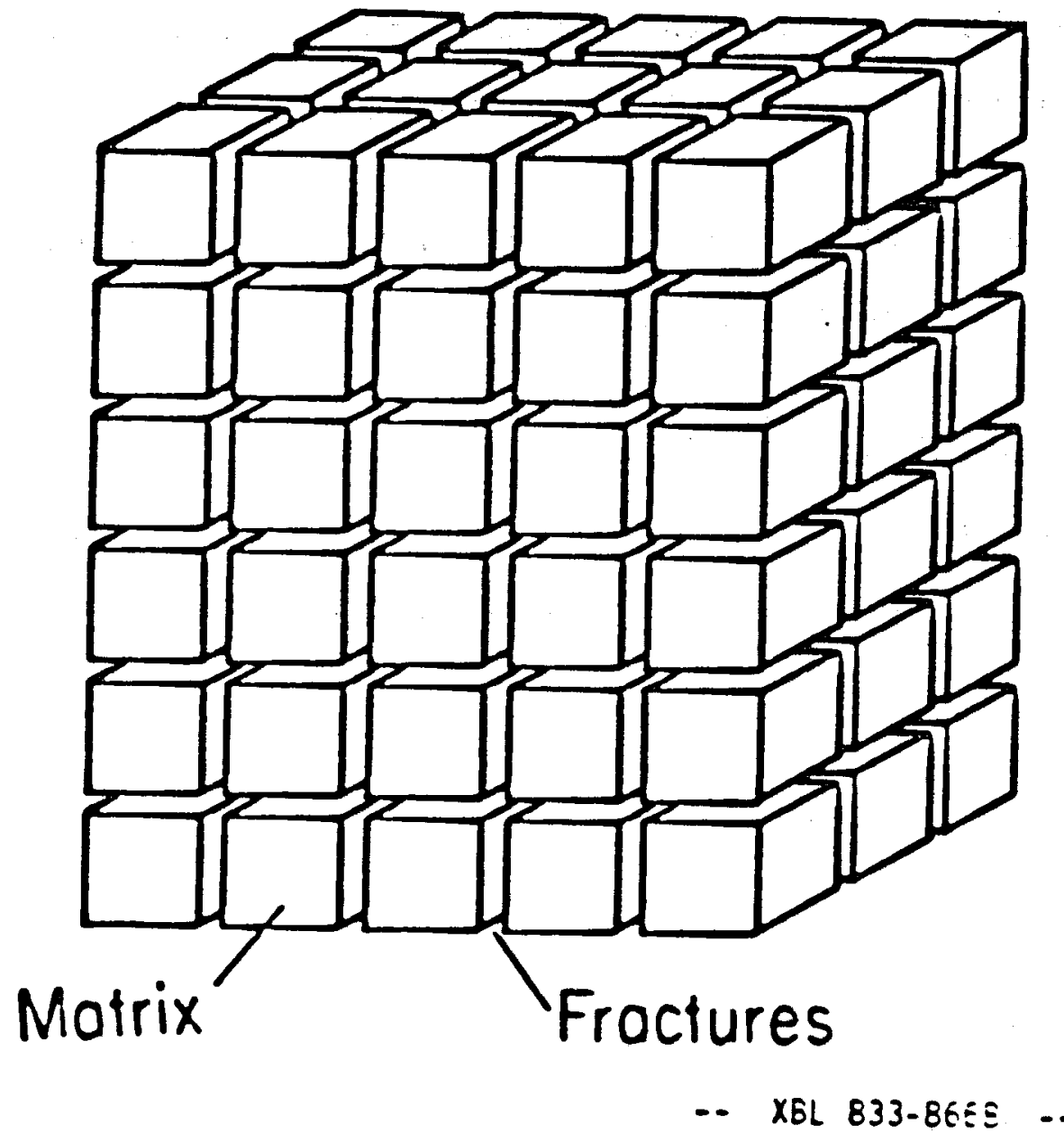

Figure 1: Idealized model of a fractured porous medium 


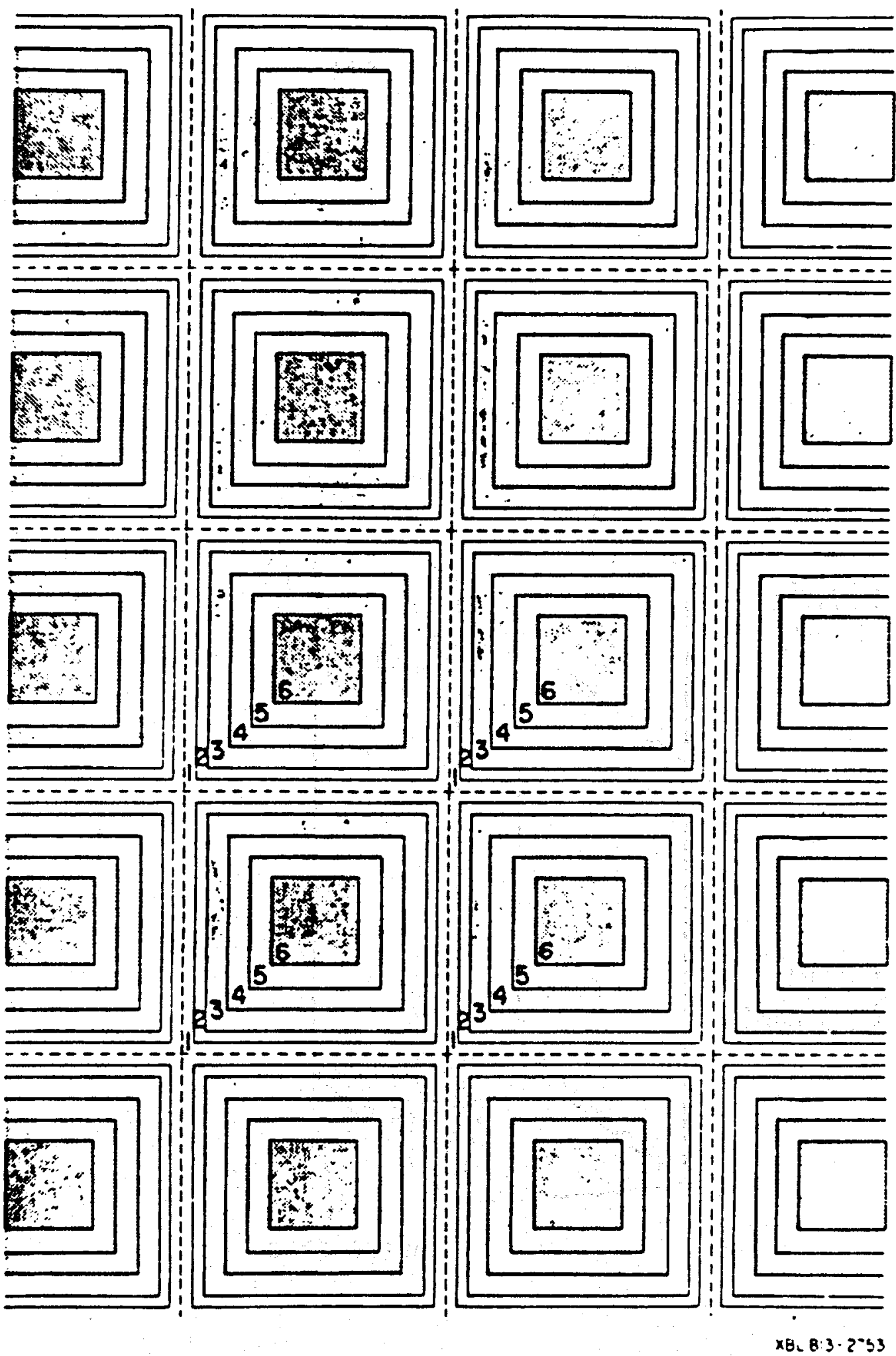

Figure 2: Computational mesh to model transport processes in fractured porous media employed by the MINC approximation 


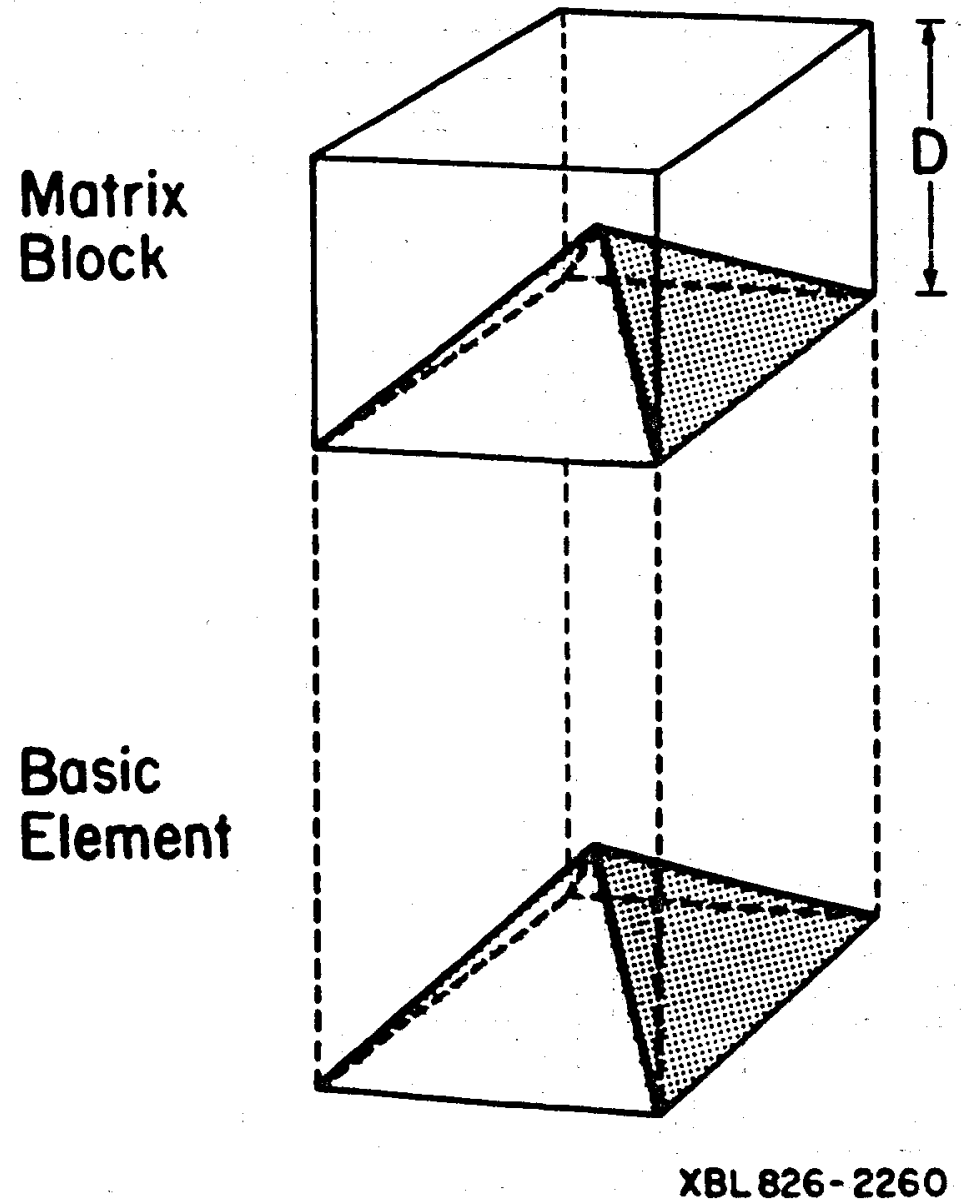

Figure 3: Basic model for a porous cube using the one-dimensional approximation 


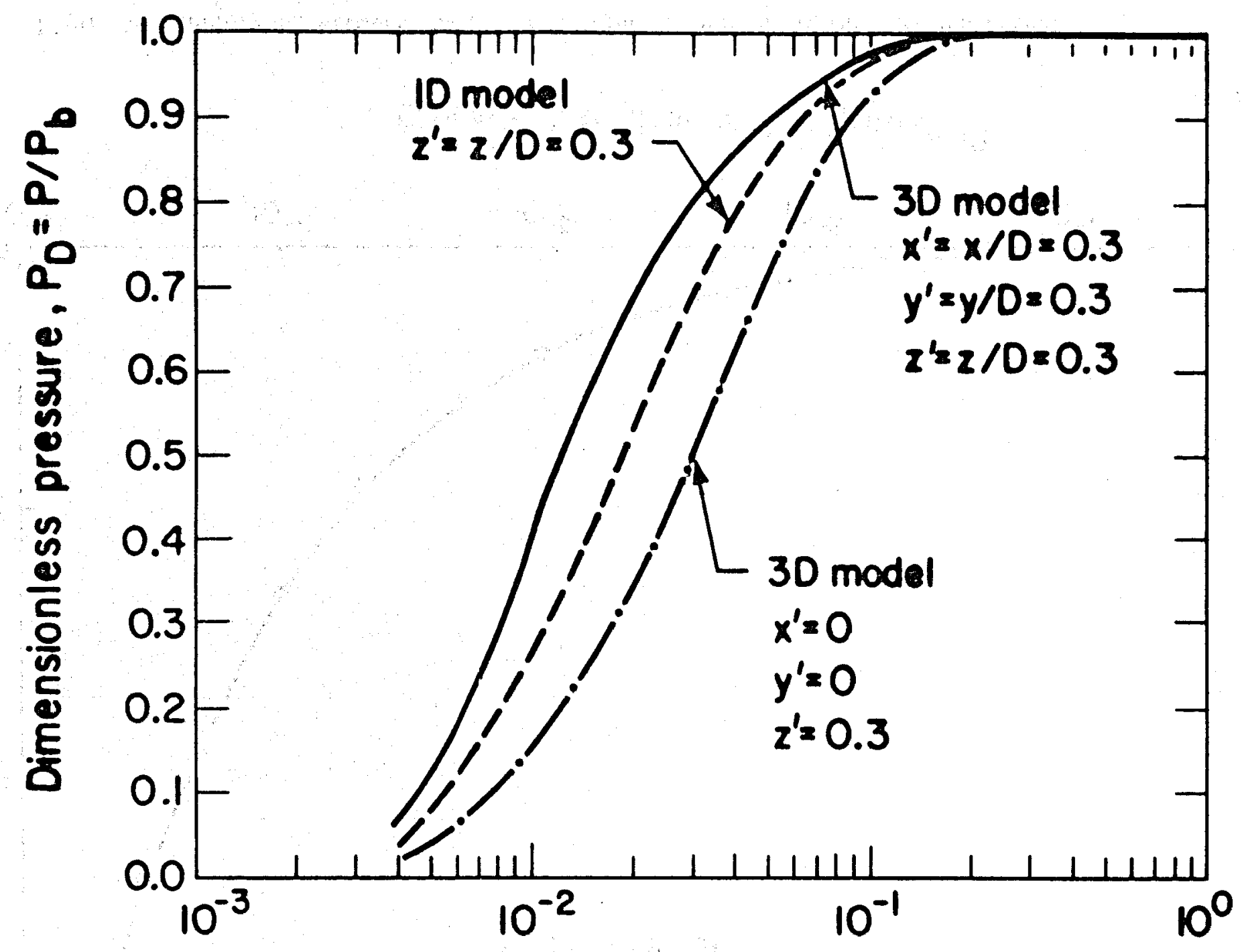

Dimensionless time, $\tau=k+/ \phi \mu c(D / 2)^{2}$

X8L835-1001

Figure 4: Pressure distributions in a porous cube calculated using the one-dimensional approximation and the exact solution (laheled 31 ) model) 


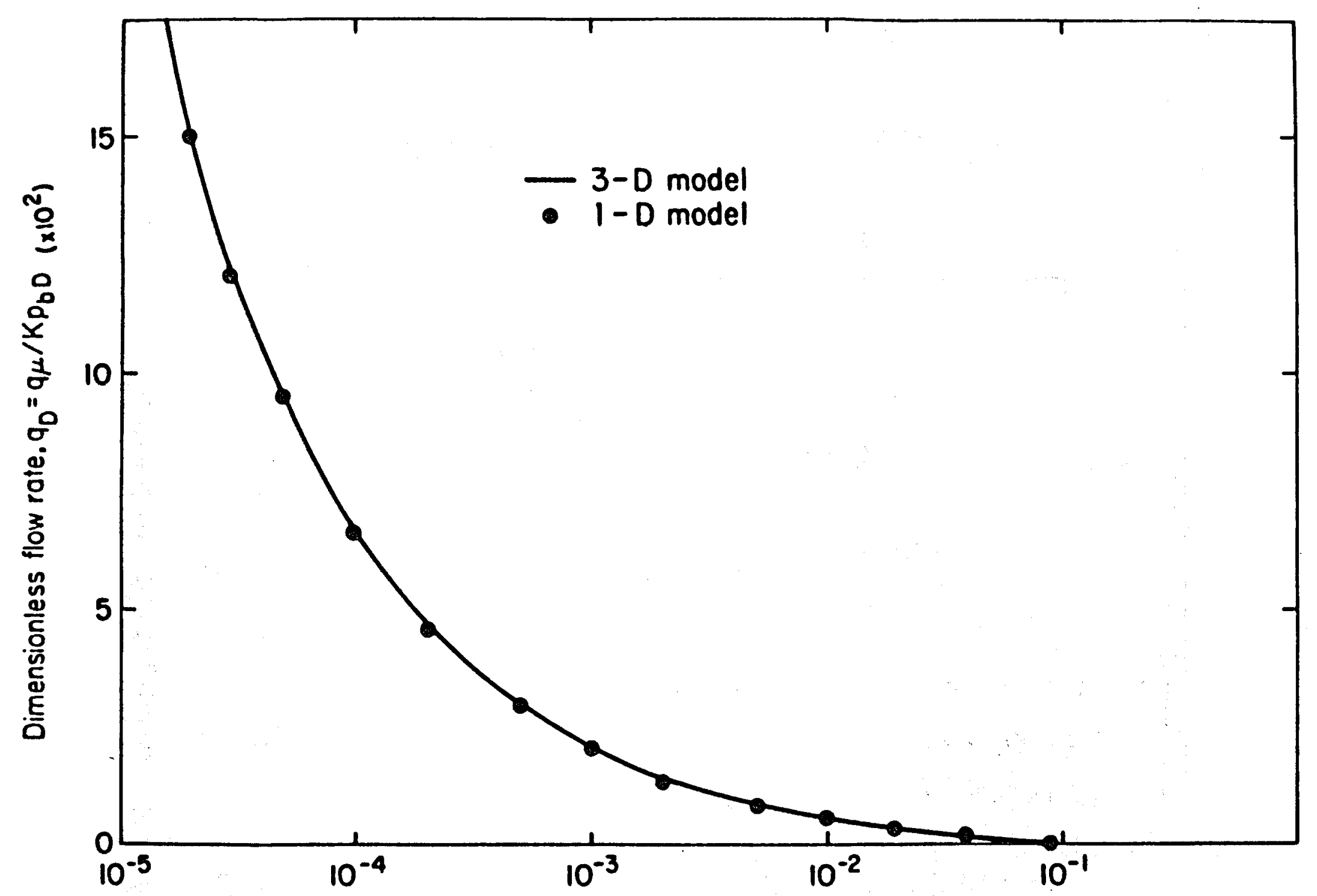

Dimensionless time, $\tau=k \uparrow / \phi \mu c(D / 2)^{2}$

X8L $825-2240$

Figure 5: Comparison between the flow rate from the cube using the one-dimensional solution and the exart solution 

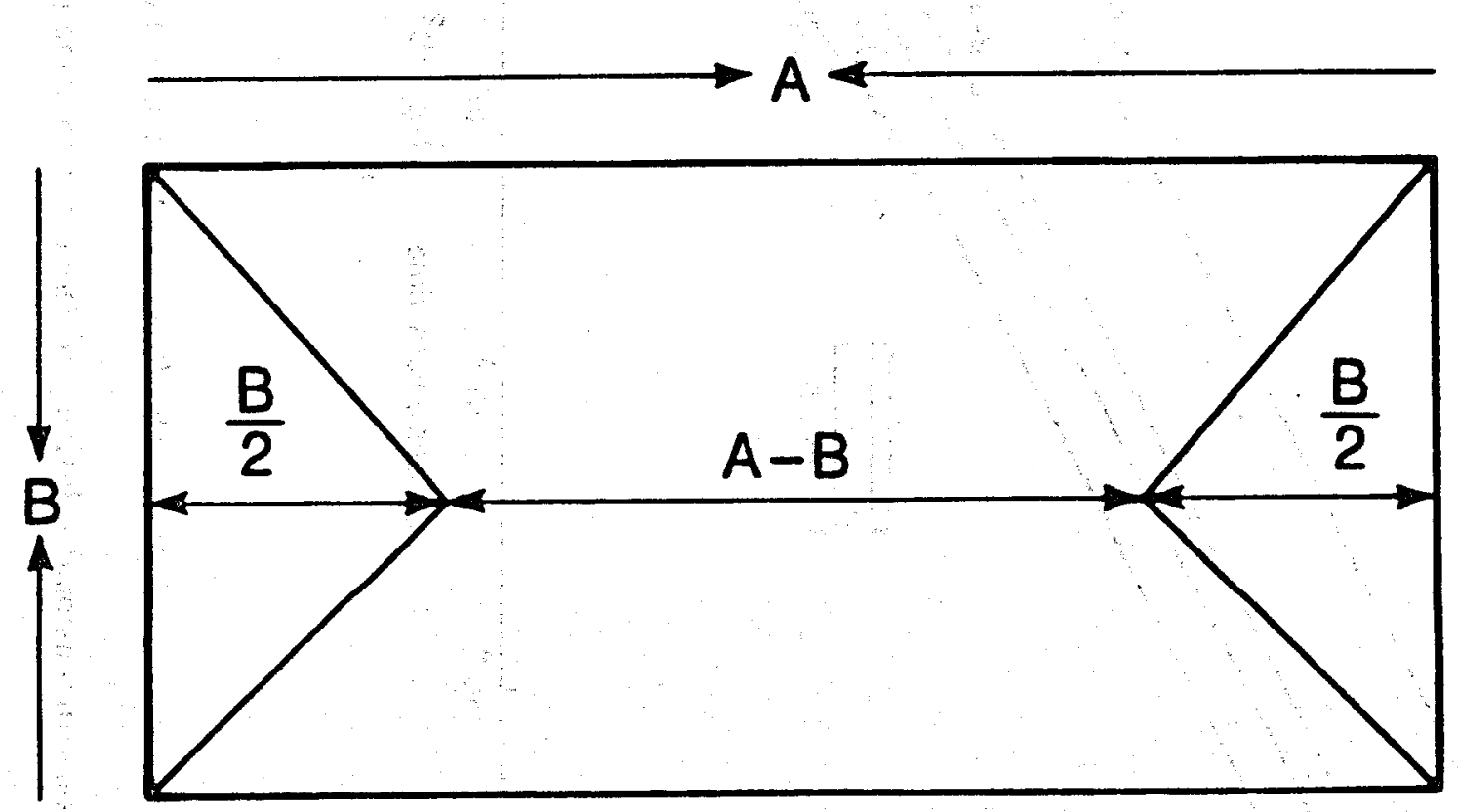

X8L 8511.11545

Figure 6: Basic model for a rectangular porous slab using the one-dimensional approximation 


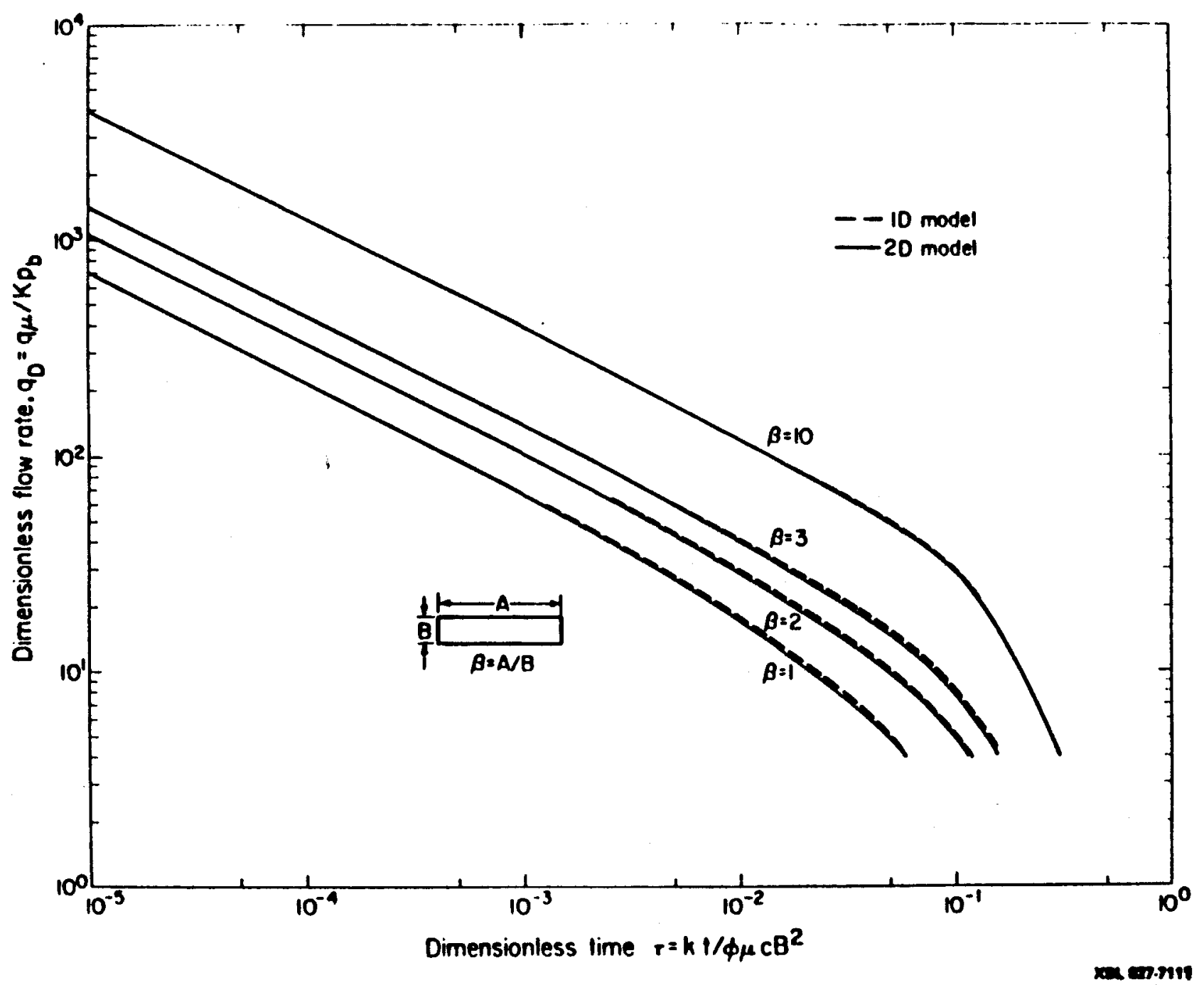

Figure 7: Comparison between the flow rate from a rectangular porous slab using the one-dimensional solution and the exact solution (labeled 2D model) 


\section{Acknowledgements}

This work was supported through U. S. Department of Energy Contract No. DC-

AC03-76SF00098 by the Assistant Secretary for Conservation and Renewable Energy, Office of Renewable Technology, Division of Geothermal Technology. 